\title{
Involvement of TRPV4 in the regulation of angiogenesis in colitis-associated cancer in mice
}

\author{
Kenjiro Matsumoto ${ }^{1}$, Daichi Utsumi ${ }^{1}$, Kikuko Amagase ${ }^{1}$, Makoto Tominaga ${ }^{2}$, Shinichi Kato ${ }^{1}$ \\ ${ }^{I}$ Department of Pharmacology and Experimental Therapeutics, Kyoto Pharmaceutical University, Japan, ${ }^{2}$ Division of \\ Cell Signaling, Okazaki Institute for Integrative Bioscience, Japan
}

Background \& Aim: Transient receptor potential vanilloid 4 (TRPV4) is a non-selective cation channel and responds to physical stimuli and various endogenous ligands such as arachidonic acid metabolites. Inflammatory bowel diseases are known as one of risk factors for colon cancer. We recently reported that the alteration of TRPV4 in vascular endothelia contributes to progression of colonic inflammation via increased vascular permeability. The present study attempts to clarify the involvement of TRPV4 in colitis-associated cancer in mice.

Methods: Eight-week-old TRPV4 deficinet (TRPV4KO) and wild-type (WT) mice were used. Colitis-associated cancer was induced by a single-azoxymethane (AOM; $12 \mathrm{mg} / \mathrm{kg}$, i.p.) injection followed by $2 \%$ dextran sulfate sodium (DSS) administration in drinking water. Survival rate, body weight, and stool score were evaluated during DSS treatment while the number and size of tumor were examined on day 56. Angiogenesis was examined by subcutaneous embedding of sponges in mice. Two weeks after subcutaneous embedding of sponges, the content of hemoglobin was determined. Immunohistochemical analyses were also performed on frozen sections of colon and sponge using various specific antibodies.

Results: AOM/DSS caused body weight loss, diarrhea, rectal bleeding, and decrease in survival rate. These responses were significantly attenuated in TRPV4KO mice when compared with WT mice. The number and size of AOM/DSS-induced tumor formation were significantly diminished in TRPV4 KO. In subcutaneous implanted sponge models, the content of hemoglobin in sponge was significantly lower in TRPV4KO than WT mice. The expression of TRPV4 was upregulated in vascular endothelia and cell-like structure in AOM/DSS-treated colon and implanted sponge. Further, the expression of TRPV4 was co-localized with CD105, a marker of angiogenesis, VEGF receptor-2, and F4/80, a marker of macrophages, in AOM/DSS-treated colon and implanted sponge. AOM/DSS-treatment and sponge implantation also upregulated the expression of CD105 and VEGFR2 in WT, but these responses were significantly attenuated in TRPV4 KO.

Conclusion: These results suggest that TRPV4 expressed in vascular endothelia and macrophages contribute to progression of colitis associated cancer via regulation of angiogenesis. 